\title{
Recuperación nutricional de lactantes marasmáticos con fórmula láctea adicionada de producto farináceo en base a lupino dulce
}

Dr. Guillermo Soza C., (1) Sr. Oskar Linn, ${ }^{(2)}$ Dr. Jaime Inostroza, (3) Dra. Helia Pettinelli L. (3) Dr. Femando Araneda L.,(3) Sra. Eugenia Saavedra R., (4) Sra. María Eugenia Gaona C.,(5) Srta. María Bosagna A. ${ }^{(6)}$

- La desnutrición infantil es uno de los grandes problemas de Salud Pública en Chile. En los sectores modestos, el acortamiento de la lactancia materna sin un adecuado sustituto en calidad y cantidad surge como origen primario del trastorno.

Por las alteraciones graves en el desarrollo de un ser en crecimiento acelerado, especialmente en el Sistema Nervioso, es la desnutrición precoz (calórico-proteica o marasmática) la que puede provocar mayor daño, potencialmente irreversible.(1-3)

Es también responsable directa o indirecta de la mayor parte de las muertes infantiles por infecciones respiratorias o digestivas. (2)

En la recuperación nutricional del lactante menor tiene importancia fundamental el aporte calórico y secundariamente el aporte proteico.(4)

La experiencia señala que estos niños pueden y deben recibir aportes de $180-200$ calorías/ kg. de peso real.(5-9)

Siendo la leche de vaca el sustituto dietético habitual de la leche humana, aparte de las modificaciones propias de la industrialización

\footnotetext{
1 Pediatra y Docente, Universidad de Chile, Sede Temuco.

2 Bioquímico, Técnico en Nutrición Instituto de Nutrición $\mathrm{N}=1$, Universidad de Giessen, Alemania Federal.

3 Pediatra S.N.S. Hospital Regional de Temuco, Chile.

${ }^{4}$ Nutricionista S.N.S. Hospital Regional de Temuco, Chile.

${ }^{5}$ Nutricionista Universidad de Chile, Sede Temuco.

${ }^{6}$ Químico-Farmacéutico. Sección Bioquímica e Inmunologia S.N.S. Hospital Regional de Temuco, Chile.
}

-pulverización, disminución de materia grasa, etc.-, el producto final, leche con materia grasa entre 12 y $26 \%$, representa un aporte proteico adecuado. Para cumplir con las exigencias dietéticas del niño con grave déficit calórico-proteico, debe ser adicionada con otros nutrientes, fundamentalmente hidratos de carbonos y/o aceites de origen vegetal, que permitan aumentar las calorías de un volumen determinado. Para estas adiciones, siempre se considera como modelo en cuanto a "proporción" de nutrientes la leche humana. ${ }^{(5-9)}$

- La asociación de harinas de cereales (trigo-cebada) con harina de leguminosas ha servido de base para múltiples mezclas, muchas de ellas actualmente en uso en países latinoamericanos. Estas mezclas no sólo aportan las calorías deficitarias, sino que proporcionan una excelente oferta proteica, ya que los aminoácidos deficitarios de las leguminosas (metionina) son complementados por las proteínas de los cereales, a la vez la lisina, generalmente limitante de los cereales, es aportada por las leguminosas.(10-15)

- Motivados por estas consideraciones, hemos diseñado una mezcla o producto farináceo mixto con ingredientes locales, que tiene como leguminosa base el lupino dulce, más harina de trigo y avena pelada, suero de leche en polvo y sacarosa en las proporciones que se aprecian en la Tabla N. ${ }^{\circ} 3$.

- Este producto se adicionó a la fórmula láctea de uso en nuestro Hospital y se decidió 
Tabla N. ${ }^{\circ} 3$

PRODUCTO FARINACEO BASE LUPINO FORMULA

\begin{tabular}{lc}
\hline \multicolumn{1}{c}{ componente } & porcentaje (g\%) \\
\hline harina de lupino & 33.3 \\
\hline harina de trigo & 16.2 \\
\hline harina de avena & 24.7 \\
\hline suero de leche en polvo & 4.3 \\
\hline sacarosa & 21.5 \\
\hline
\end{tabular}

utilizarlo en la realimentación de lactantes marasmáticos.

- El lupino dulce se cultiva desde Bío-Bío hasta Llanquihue, siendo la IX Región de alto rendimiento y proyección agronómica. Numerosos y documentados estudios muestran su excelente tenor proteico (sobre $40 \%$ ) y de materia grasa $(16 \%)$ con riqueza de ácidos grasos no saturados. (16-25)

Por estas razones y por haberse cumplido ya las etapas previas que señalan su inocuidad toxicológica en el ser humano, es que se consideró como ingrediente fundamental de la mezcla referida.

\section{OBJETIVOS}

1) Obtener la recuperación nutricional de los lactantes marasmáticos en base a una fórmula láctea enriquecida con producto farináceo mixto (leche-lupino).

2) Medir la aceptabilidad y tolerancia de esta mezcla, en base a parámetros clínicos y bioquímicos.

3) Analizar los aspectos cuali-cuantitativos de esta recuperación, también en relación a indicadores clínicos y de laboratorio, con patrones comparativos considerados normales y consigo mismo.

4) Introducir nuevos alimentos calórico-proteicos en la alimentación del niño y adulto normal y desnutrido, en base a proteínas vegetales no convencionales.

5) Contribuir a la reparación nutricional infantil individual y colectiva con,

6) la utilización de recursos dietéticos de origen nacional y regional, con las proyecciones de tipo económico y social consiguientes.

\section{MATERIAL Y METODO}

- Estudio efectuado en el Servicio de Pediatría del Hospital Regional de Temuco, entre septiembre de 1977 y abril de 1978.

Se alimentaron diez lactantes cuyas edades oscilaban entre tres meses y un año de edad, portadores de desnutrición calórico-proteica grave - Grados II y III- aplicando tablas de crecimiento para edad y talla de Sempé y usando el criterio de Gómez.(26, 27)

- Estos lactantes fueron captados en Policlínico de Desnutridos y no presentaban patología asociada o intercurrente.

- Cada niño recibió, durante tres meses, la fórmula láctea habitual utilizada en el Servicio de Pediatría con adición del producto farináceo al 5\% (Tablas Nos. 4, 5, 6). El producto se pre-

Tabla N. ${ }^{\circ} 4$

PRODUCTO FARINACEO BASE LUPINO DULCE

Análisis nutritivo de los diferentes componentes de la mezcla

Proteina Aceite H. de C.

\begin{tabular}{llll}
\hline $\begin{array}{c}\text { Harina de } \\
\text { lupino } \\
\text { seca }\end{array}$ & 38.1 & 16.1 & 9.0 \\
\hline $\begin{array}{c}\text { Harina de } \\
\text { trigo } \\
\text { precocida }\end{array}$ & 17.3 & 3.9 & 60.0 \\
\hline $\begin{array}{c}\text { Harina de } \\
\text { avena } \\
\text { precocida }\end{array}$ & 16.8 & 7.6 & 49.0 \\
\hline $\begin{array}{c}\text { Suero } \\
\text { de } \\
\text { leche }\end{array}$ & 1.2 & 2.7 & 5.0 \\
\hline
\end{tabular}

Tabla N. ${ }^{\circ} 5$

PRODUCTO FARINACEO BASE LUPINO

Análisis nutritivo

\begin{tabular}{lc}
\hline Proteína $(\mathrm{N} \times 6.25)$ & $19.7 \%$ \\
\hline Grasa & $8.0 \%$ \\
\hline Fibra & $2.3 \%$ \\
\hline Carbohidrato & $43.5 \%$ \\
\hline Calorias & $345.8 \%$ \\
\hline
\end{tabular}


sentaba en forma de un polvo fino de color amarillo-café, sin mal olor y de fácil disolución.

- La preparación se efectuó en forma estrictamente rigurosa por personal del Servicio Dietético Infantil (SEDILE). Tiempo de cocción, 10 minutos. Esterilización terminal.

-En las Tablas N. ${ }^{\circ}$ s 5 y 6 se aprecia el valor nutritivo de la mezcla y en la Tabla N. ${ }^{\circ} 14$ el análisis de los aminoácidos de los diferentes componentes del producto.

- Se efectuó análisis diario de ingesta calórica y proteica, aumentando progresivamente el aporte, (5-7) para alcanzar entre la segunda y tercera semana de observación cifras de 180-200 cal./kg. peso real.

En todos los casos se realizó evaluación antropométrica rigurosa estandarizada con determinación periódica de peso, talla y perímetro craneano.
- Se determinaron los siguientes parámetros de laboratorio:

1) Hemograma completo (inicial y final).

2) Proteinemia: fracción Albúmina y Globulina -por el método de Biuret modificado.

3) Inmunoglobulinasséricas (IgA - IgH - IgG) Técnica de inmunodifusión radial.

4) Urea sanguínea.

5) Transaminasas (Glutámica-oxaloacética (GOA) y Glutámica Pirúvica (GP)). Método de Gootmann y Frankel.

6) Creatinina Urinaria.

- En todos los niños se efectuó estimulación sicomotora por personal especializado (Kinesiólogo - Educadora de Párvulos).

Posterior al alta se continuó el control del niño y núcleo familiar por Equipo de Nutrición en Consultorio Externo.

Tabla N. ${ }^{\circ} 6$

FORMULA LACTEA ADICIONADA CON PRODUCTO FARINACEO BASE - LUPINO DULCE

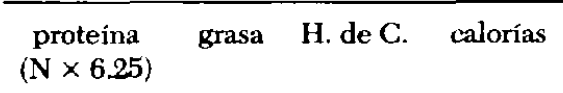

\begin{tabular}{|c|c|c|c|c|}
\hline $\begin{array}{l}\text { FORMULA } \\
\text { HABITUAL } \\
\text { leche } 18 \% \\
\text { materia grasa } \\
\text { al } 10 \%\end{array}$ & 3.2 & 1.8 & 4 & 46 \\
\hline $\begin{array}{l}\text { SACAROSA } \\
\text { al } 5 \%\end{array}$ & - & - & 5 & 20 \\
\hline $\begin{array}{l}\text { PRODUCTO } \\
\text { FARINACEO } \\
\text { al } 5 \%\end{array}$ & 1 & 0.5 & 2.2 & 18 \\
\hline TOTAL & 4.2 & 2.3 & 11.2 & 84 \\
\hline
\end{tabular}

Tabla N. ${ }^{\circ} 7$

GRADO DE DESNUTRICION Y GANANCIA PONDERAL DIARIA PROMEDIO EN 10 LACTANTES MARASMATICOS TRATADOS

\begin{tabular}{|c|c|c|c|c|}
\hline $\begin{array}{l}\text { CASO } \\
\text { N. }\end{array}$ & $\begin{array}{l}\text { EDAD } \\
\text { INICIAL }\end{array}$ & $\begin{array}{c}\text { GRADO } \\
\text { DESNUTHIC. }\end{array}$ & $\begin{array}{l}\text { INCREM. } \\
\text { DIARIO }\end{array}$ & $\begin{array}{l}\text { PROMEDIO } \\
\text { NORMAL }\end{array}$ \\
\hline 1 & $9 \mathrm{~ms}$ & III & 42.5 & 10 \\
\hline 2 & $2 \mathrm{~ms} .23 \mathrm{ds}$. & III & 30.7 & 25 \\
\hline 3 & $3 \mathrm{~ms} .20 \mathrm{ds}$. & II - III & 26.1 & 20 \\
\hline 4 & $7 \mathrm{~ms}$ & III & 23.0 & 15 \\
\hline 5 & $4 \mathrm{~ms} .5 \mathrm{ds}$. & III & 31.0 & 20 \\
\hline 6 & $4 \mathrm{~ms}$ & III & 40.0 & 20 \\
\hline 7 & $3 \mathrm{~ms} .11 \mathrm{ds}$. & II - III & 32.0 & 20 \\
\hline 8 & $5 \mathrm{~ms} .20 \mathrm{ds}$. & III & 26.0 & 15 \\
\hline 9 & $3 \mathrm{~ms}$ & II - III & 40.0 & 20 \\
\hline 10 & $6 \mathrm{~ms} .9 \mathrm{ds}$. & II - III & 39.0 & 20 \\
\hline
\end{tabular}




\section{RESULTADOS}

— En la Tabla N. 7 y Gráfico N. ${ }^{\circ} 1$ se aprecia el incremento ponderal de los diez niños.

Se compara el promedio diario de ganancia ponderal con los valores correspondientes a su edad. En todos hubo un aumento de peso diario mayor que lo normal, que en ocasiones cuadruplica la velocidad de crecimiento propia de su grupo etario. $(26,27)$
- En la Tabla N. ${ }^{\circ} 8$ se observan los valores iniciales y finales de talla y la excreción de creatinina urinaria. Se expresa la relación o índice Creatinina/Talla, el que aumenta en todos los niños bajo tratamiento nutricional.

- La Tabla N. 9 muestra los valores Proteína-Albúmina y globulina-sanguínea inicial, media y final. En todos los casos se encontraron valores bajos de proteína total, a expensas de albúmina, los que se corrigen en el curso de los

Tabla N. ${ }^{\circ} 8$

VALORES INICLALES Y FINALES DE TALLA, CREATININA URINARIA e INDICE CREATININA/TALLA PARA 10 LACTANTES DESNUTRIDOS BAJO TRATAMIENTO

\begin{tabular}{|c|c|c|c|c|c|c|c|}
\hline \multirow[t]{2}{*}{$\begin{array}{l}\text { CASO } \\
\text { N. }\end{array}$} & \multirow[t]{2}{*}{ EDAD } & \multicolumn{2}{|c|}{$\begin{array}{l}\text { TALLA } \\
\mathrm{cm} .\end{array}$} & \multicolumn{2}{|c|}{$\begin{array}{l}\text { CREATININA } \\
\mathrm{mg} / 24 \text { horas }\end{array}$} & \multicolumn{2}{|c|}{$\begin{array}{c}\text { INDICE } \\
\text { creatinina/talla } \\
\mathrm{mg} / \mathrm{cm} / 24 \text { horas }\end{array}$} \\
\hline & & inicio & término & inicio & término & inicio & término \\
\hline 1 & $9 \mathrm{~ms}$ & 62 & 69 & 470 & 1020 & 8 & 14 \\
\hline 2 & $2 \mathrm{~ms} .23 \mathrm{ds}$ & 55 & 63 & 360 & 1200 & 7 & 20 \\
\hline 3 & $3 \mathrm{~ms} .20 \mathrm{ds}$ & 56 & 64 & 690 & 1500 & 17 & 25 \\
\hline 4 & $7 \mathrm{~ms}$ & 66 & 72 & 460 & 950 & 8 & 13 \\
\hline 5 & $4 \mathrm{~ms} .5 \mathrm{ds}$. & 54 & 63 & 380 & 1260 & 6 & 20 \\
\hline 6 & $4 \mathrm{~ms}$ & 51 & 56 & 400 & 1060 & 8 & 18 \\
\hline 7 & $3 \mathrm{~ms} .11 \mathrm{ds}$ & 60 & 68 & 410 & 920 & 7 & 15 \\
\hline 8 & $5 \mathrm{~ms} .20 \mathrm{ds}$ & 56 & 66 & 680 & 1120 & 12 & 20 \\
\hline 9 & $3 \mathrm{~ms}$ & 54 & 60 & 920 & 1580 & 17 & 20 \\
\hline 10 & $6 \mathrm{~ms} .9 \mathrm{ds}$. & 59 & 63 & 1120 & 1630 & 18 & 25 \\
\hline
\end{tabular}

Tabla N. 9

VALORES SANGUINEOS DE PROTEINA - ALBUMINA - GLOBULINA EN 10 LACTANTES MARASMATICOS EN RECUPERACION NUTRICIONAL

\begin{tabular}{|c|c|c|c|c|c|c|c|c|c|}
\hline \multirow{2}{*}{$\begin{array}{l}\text { CASO } \\
\text { N. }\end{array}$} & \multicolumn{3}{|c|}{$\begin{array}{c}\text { PROTEINA } \\
\mathrm{g} \%\end{array}$} & \multicolumn{3}{|c|}{$\begin{array}{c}\text { ALBUMINA } \\
g \%\end{array}$} & \multicolumn{3}{|c|}{$\begin{array}{c}\text { GLOBULINA } \\
\mathrm{g} \%\end{array}$} \\
\hline & $1 .^{\circ}$ & $2 .^{\circ}$ & $3 .^{\circ}$ & $1 .^{\circ}$ & $20^{\circ}$ & $3 .^{\circ}$ & $10^{\circ}$ & $2 .^{\circ}$ & $30^{\circ}$ \\
\hline 1 & 5.6 & 6.3 & 7.2 & 2.5 & 3.0 & 4.1 & 3.1 & 3.3 & 3.1 \\
\hline 2 & 4.6 & 5.8 & 6.8 & 2.2 & 2.8 & 3.5 & 2.4 & 3.0 & 3.3 \\
\hline 3 & 5.0 & 5.8 & 6.5 & 3.0 & 3.6 & 4.1 & 2.0 & 2.2 & 2.4 \\
\hline 4 & 4.9 & 5.8 & 6.9 & 2.4 & 3.2 & 4.0 & 2.5 & 2.6 & 2.8 \\
\hline 5 & 4.8 & 5.4 & 6.0 & 2.5 & 2.9 & 3.2 & 2.3 & 2.5 & 2.8 \\
\hline 6 & 4.6 & 5.4 & 6.5 & 2.4 & 3.0 & 3.8 & 2.2 & 2.4 & 2.7 \\
\hline 7 & 5.6 & 6.5 & 7.6 & 2.8 & 3.4 & 4.2 & 2.8 & 3.1 & 3.4 \\
\hline 8 & 4.5 & 5.4 & 6.6 & 2.3 & 3.1 & 3.9 & 2.2 & 2.3 & 2.7 \\
\hline 9 & 4.8 & 6.0 & 6.9 & 2.9 & 3.9 & 4.8 & 1.7 & 2.1 & 2.1 \\
\hline 10 & 4.6 & 5.8 & 6.9 & 2.3 & 3.0 & 4.1 & 2.3 & 2.8 & 2.8 \\
\hline
\end{tabular}

valores normales

6.4 a $8 \mathrm{~g} \%$

3.8 a $4.7 \mathrm{~g} \%$

2.3 a $4.1 \mathrm{~g} \%$ 
GRAFICO N $^{\circ} 1$

INCREMENTO PODERAL EN 10 LACTANTES

MARASMATICOS

EXPERIENCIA PRODUCTO BASE LUPINO DULCE

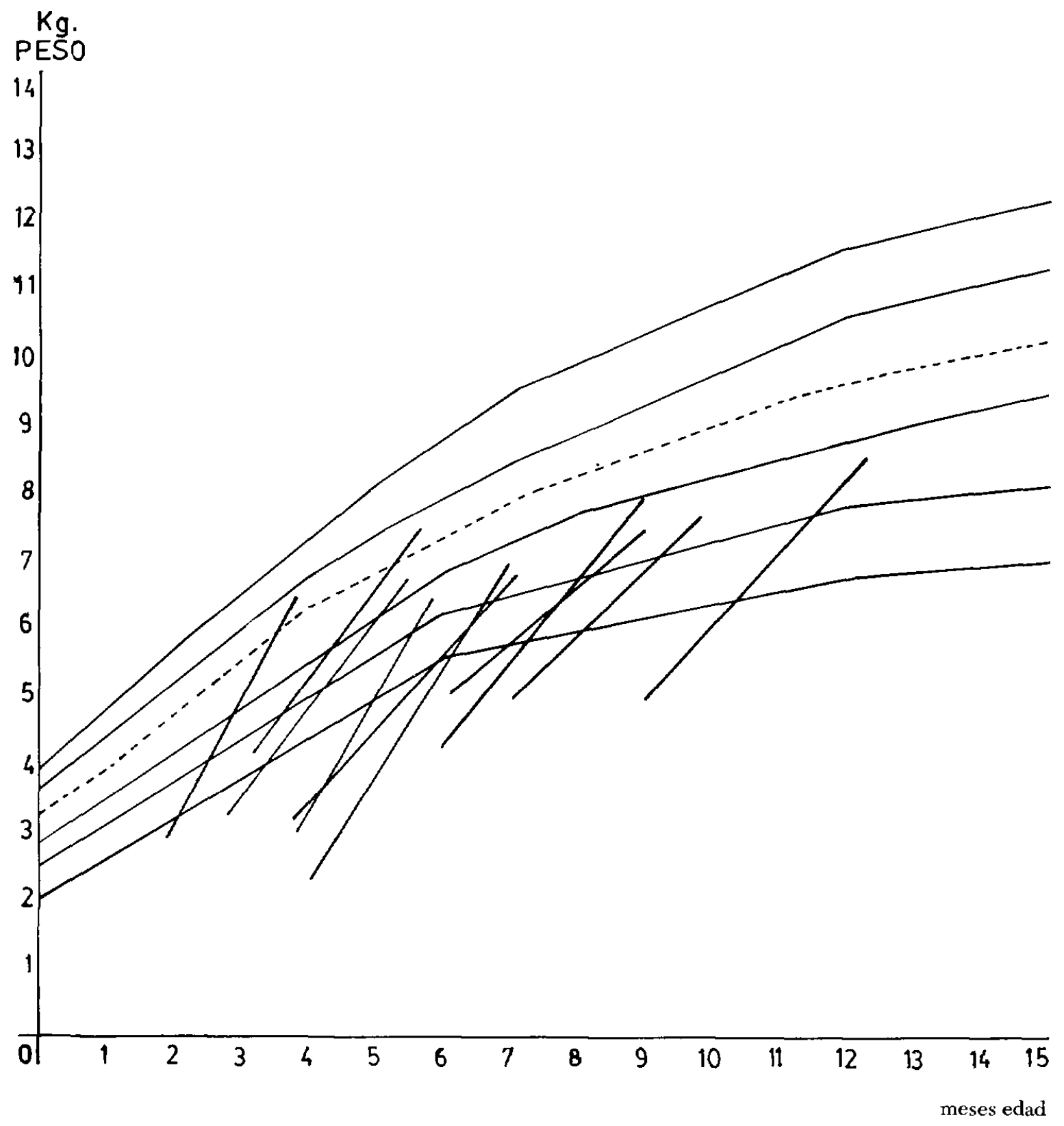

Adaptado de Sempé y colab. 
tres meses de tratamiento en todos los casos. Las cifras de globulinas permanecen en cambio más constantes.

- En las Tablas Nos. 10 y 11 se observan los valores de Transaminasas y de Urea sanguínea al inicio y al término de 3 meses de tratamiento nutricional.

Se puede apreciar que los valores mantienen su normalidad.
- Las Inmunoglobulinas muestran valores ligeramente menores al ingreso (Tabla N. ${ }^{\circ} 11$ ), siempre dentro de los rangos de normalidad proporcionados por el Instituto Bacteriológico, Sección Inmunología - para este grupo etario-. Después de la recuperación dietética se observan valores mayores, pero siempre en límites de normalidad.

Tabla N. ${ }^{\circ} 10$

VALORES DE TRANSAMINASAS EN 10 LACTANTES

MARASMATICOS EN RECUPERACION NUTRICIONAL

TRANSAMINASAS

\begin{tabular}{|c|c|c|c|c|c|c|}
\hline \multirow{2}{*}{$\begin{array}{c}\text { CASO } \\
\text { N. }\end{array}$} & \multicolumn{3}{|c|}{ S G P T $(\mathrm{U} / \mathrm{ml})$} & \multicolumn{3}{|c|}{$\mathrm{S} \mathrm{GOT}(\mathrm{U} / \mathrm{ml})$} \\
\hline & $1 .^{o}$ & $2 .^{\circ}$ & $3 .^{\circ}$ & $1 .^{o}$ & $2 .^{\circ}$ & 3. ${ }^{\circ}$ \\
\hline 1 & 14 & 15 & 14 & 35 & 20 & 25 \\
\hline 2 & 13 & 19 & 25 & 20 & 21 & 30 \\
\hline 3 & 13 & 12 & 14 & 26 & 30 & 24 \\
\hline 4 & 15 & 28 & 25 & 35 & 30 & 32 \\
\hline 5 & 13 & 35 & 25 & 35 & 20 & 35 \\
\hline 6 & 19 & 32 & 17 & 18 & 19 & 22 \\
\hline 7 & 22 & 24 & 22 & 15 & 17 & 22 \\
\hline 8 & 25 & 28 & 26 & 25 & 18 & 30 \\
\hline 9 & 28 & 30 & 27 & 24 & 35 & 34 \\
\hline 10 & 13 & 19 & - & 39 & 41 & - \\
\hline $\begin{array}{c}\text { valor } \\
\text { normal }\end{array}$ & \multicolumn{3}{|c|}{$5-30$} & \multicolumn{3}{|c|}{$8-40$} \\
\hline
\end{tabular}

Tabla N. ${ }^{\circ} 11$

VALORES SANGUINEOS DE INMUNOGLOBULINAS EN 10 LACTANTES MARASMATICOS EN RECUPERACION NUTRICIONAL

INMUNOGLOBULINAS (mg\%)

\begin{tabular}{|c|c|c|c|c|c|c|c|c|c|}
\hline \multirow{2}{*}{$\begin{array}{c}\text { CASO } \\
\text { N. }^{-}\end{array}$} & \multicolumn{3}{|c|}{ A (Iga) } & \multicolumn{3}{|c|}{$\mathrm{G}(\mathbf{I g} \mathrm{G})$} & \multicolumn{3}{|c|}{$\mathbf{M}(\mathbf{I g M})$} \\
\hline & $1 .^{o}$ & $20^{\circ}$ & $3 .^{\circ}$ & $1 .^{\circ}$ & $20^{\circ}$ & $3 .^{\circ}$ & $1 .^{\circ}$ & $2 .^{\circ}$ & $3 .^{\circ}$ \\
\hline 1 & 150 & 160 & 190 & 700 & 740 & 860 & 135 & 220 & 300 \\
\hline 2 & 155 & 220 & 180 & 1260 & 1540 & 1550 & 160 & 210 & 200 \\
\hline 3 & 125 & 160 & 215 & 620 & 1140 & 1540 & 175 & 250 & 280 \\
\hline 4 & 250 & 150 & 180 & 550 & 920 & 1480 & 135 & 145 & 160 \\
\hline 5 & 135 & 180 & 165 & 1100 & 1420 & 1600 & 120 & 200 & 240 \\
\hline 6 & 100 & 120 & 195 & 1240 & 1720 & 2150 & 82 & 165 & 205 \\
\hline 7 & 125 & 215 & 235 & 1100 & 1810 & 1850 & 155 & 200 & 200 \\
\hline 8 & 145 & 155 & 215 & 1100 & 1280 & 1940 & 155 & 162 & 210 \\
\hline 9 & 350 & 300 & 390 & 1840 & 2250 & 2450 & 210 & 250 & 250 \\
\hline 10 & 170 & 245 & - & 1350 & 1750 & - & 150 & 230 & - \\
\hline $\begin{array}{c}\text { valor } \\
\text { normal }\end{array}$ & \multicolumn{3}{|c|}{$104-240$} & \multicolumn{3}{|c|}{$600-1200$} & \multicolumn{3}{|c|}{ Gr. 193} \\
\hline
\end{tabular}


- Finalmente en la Tabla $N .^{\circ} 12$ se señalan las cifras iniciales y finales para Hemoglobina $y$ Hematocrito en los diez pacientes.

En el primer semestre de vida se presenta la anemia fisiológica, más precoz en los prematuros.

Se consideran "cifras críticas" las de $10 \mathrm{gr} . \%$ para Hemoglobina y de $30 \%$ para Hematocrito. Considerando estas cifras convencionales, los pacientes 2, 4, 7 y 9 presentaban las cifras más bajas que permite señalarlas como patológicas. Se puede apreciar en todos los casos mejoría de estos parámetros, con valores estacionarios para los pacientes 1 y 5 .

\section{DISCUSION}

- El análisis de los resultados obtenidos nos permite señalar que la recuperación de los diez lactantes desnutridos ha sido satisfactoria, tanto desde el punto de vista clínico como de los indicadores bioquímicos. La aceptabilidad y tolerancia de la fórmula láctea ha sido muy buena, sin presentar trastornos digestivos ni signos de intolerancia o compromisos en parénquimas hepático o renal (clínico o de laboratorio).
- Incremento Ponderal:

Los incrementos señalados son satisfactorios. Es conocido(4-8) el hecho que si a un lactante desnutrido se le proporcionan las calorías y proteínas deficitarias más los requerimientos normales, no sólo reanuda, sino que sobrepasa la velocidad de crecimiento normal, considerándose como optimos valores de 30-40 gr./día, que sólo los tiene el niño sano en el primer trimestre de vida. Cualquier fórmula láctea balanceada, con aportes calóricos proteicos que cubran los factores señalados (Tablas Nos. 1 y 2 ) nos llevaría al mismo resultado, siempre que no presente enfermedades intercurrentes.

Nuestros lactantes recibieron aportes progresivos de la fórmula mencionada, superando en algunos casos las $200 \mathrm{cal} / \mathrm{kg}$ (alimentados adlibitum).

El gráfico muestra en los trazos la unión de valores iniciales y finales de peso. Se aprecia en todos mejoría del grado de desnutrición en los tres meses que duró la experiencia, con cinco niños eutróficos y cinco en grado leve de desnutrición al término del trimestre.

Tabla N. ${ }^{0} .12$

VALORES SANGUINEOS DE HEMOGLOBINA Y HEMATOCRITO EN 10 LACTANTES MARASMATICOS EN RECUPERACION NUTRICIONAL

\begin{tabular}{rlrccc}
\hline $\begin{array}{c}\text { CASO } \\
\text { N. }\end{array}$ & \multicolumn{1}{c}{$\begin{array}{c}\text { EDAD } \\
\text { INGRESO }\end{array}$} & $\begin{array}{c}\text { VALORES } \\
\text { HG }(\mathrm{g} \%)\end{array}$ & $\begin{array}{c}\text { INICLALES } \\
\text { HCTO }(\%)\end{array}$ & $\begin{array}{c}\text { VALORES } \\
\text { HG }(\mathrm{g} \%)\end{array}$ & HCTO (\%) \\
\hline 1 & $9 \mathrm{~ms}$. & 11.8 & 35 & 11.2 & 32 \\
2 & $2 \mathrm{~ms} .23 \mathrm{ds}$. & 9.8 & 29 & 14.5 & 38 \\
3 & $3 \mathrm{~ms} .20 \mathrm{ds}$. & 11.5 & 36 & 13.2 & 39 \\
4 & $7 \mathrm{~ms}$. & 7.4 & 28 & 11.2 & 35 \\
5 & $4 \mathrm{~ms} .5 \mathrm{ds}$. & 11.3 & 34 & 11.5 & 35 \\
6 & $4 \mathrm{~ms}$. & 11.5 & 34 & 12.5 & 38 \\
7 & $3 \mathrm{~ms} .11 \mathrm{ds}$. & 10 & 30 & 11.2 & 33 \\
8 & $5 \mathrm{~ms} .11 \mathrm{ds}$. & 11.5 & 37 & 12.5 & 38 \\
9 & $3 \mathrm{~ms}$. & 9.5 & 29 & 10.9 & 32 \\
10 & $6 \mathrm{~ms} .9 \mathrm{ds}$. & 10.5 & 32 & 12.3 & 35 \\
\hline
\end{tabular}

Tabla N. ${ }^{\circ} 1$

RECOMENDACIONES CALORICAS

ler. AÑO DE VIDA

\begin{tabular}{cc}
\hline Edad & Calorías/kg. peso/día \\
\hline $10^{\circ}-3$ er. mes & 120 \\
\hline 3 er. $-9 .^{\circ}$ mes & 110 \\
\hline $9 .^{\circ}-12 .^{\circ}$ mes & 100 \\
\hline
\end{tabular}


Tabla N. ${ }^{\circ}$

RECOMENDACIONES DE PROTEINAS EN

LACTANTES MENORES NORMALES

\begin{tabular}{ccc}
\hline $\begin{array}{l}\text { Edad } \\
\text { (meses) }\end{array}$ & $\begin{array}{c}\text { Ingesta del lactante } \\
\text { alimentado a pecho } \\
\mathrm{g} / \mathrm{kg} / \text { día }\end{array}$ & Recomendación \\
\hline 1 & 2.2 & 3.0 \\
\hline 2 & 2.0 & 2.7 \\
\hline 4 & 1.7 & 2.3 \\
\hline 6 & 1.6 & 2.2 \\
\hline 8 & 1.3 & 1.8 \\
\hline 10 & 1.2 & 1.6 \\
\hline 12 & 1.2 & 1.6 \\
\hline
\end{tabular}

Fuente: FAO/WHO 1976.

- En relación al aporte proteico, de la Tabla N. ${ }^{\circ} 6$ se deduce que las calorías aportadas por las proteínas no sobrepasan un $19 \%$, con lo que se mantiene una relación Calorías Totales/Calorías Proteicas, similar a las fórmulas lácteas enriquecidas con alimentos en uso en nuestra población de desnutridos. Si se observan los alimentos pro- teicos proporcionados por el Servicio Nacional de Salud, a través del Convenio con la Obra Filantrópica Adventista OFASA (Tabla N. ${ }^{\circ}$ 14), que se usan en concentraciones de hasta el $10 \%$, vemos que el $\mathrm{P} \%$ de estas fórmulas es superior.

- Sin lugar a dudas que la mejor utilización de esta mezcla farinácea proteica balanceada

Tabla N. ${ }^{0} 14$

ANALISIS DE AMINOACIDOS

TRABAJO PRODUCTO LUPINO

\begin{tabular}{ccccc}
\hline AA & $\begin{array}{c}\text { Harina de Lupino } \\
\text { tostada } \\
\text { lupinus albus }\end{array}$ & $\begin{array}{c}\text { Harina de Trigo } \\
\text { tostada }\end{array}$ & $\begin{array}{c}\text { Harina de Avena } \\
\text { tostada }\end{array}$ & $\begin{array}{c}\text { Suero } \\
\text { en } \\
\text { polvo }\end{array}$ \\
\hline ASP & 10.4 & 5.2 & 8.1 & 9.2 \\
THR & 3.8 & 3.1 & 3.7 & 7.0 \\
SER & 6.0 & 5.2 & 4.8 & 5.6 \\
GLU & 20.5 & 27.8 & 19.4 & 15.8 \\
PRO & 4.7 & 8.6 & 4.1 & 6.9 \\
GLY & 4.4 & 4.2 & 5.1 & 2.5 \\
ALA & 3.8 & 4.0 & 4.5 & 4.8 \\
CYS & 0.9 & 1.6 & 2.0 & 1.7 \\
VAL & 4.3 & 4.1 & 5.0 & 5.9 \\
MET & 0.3 & 1.1 & 1.1 & 2.1 \\
ILE & 4.8 & 3.7 & 4.2 & 5.6 \\
LEU & 7.8 & 6.7 & 7.1 & 9.4 \\
TYR & 4.8 & 3.4 & 4.0 & 2.9 \\
PHE & 3.6 & 4.1 & 4.8 & 2.8 \\
LYS & 5.0 & 2.6 & 3.7 & 5.1 \\
HIS & 2.7 & 2.6 & 2.5 & 2.2 \\
ARG & 10.9 & 5.3 & 7.5 & 2.6 \\
TRY & 0.9 & 1.3 & 1.0 & 3.2 \\
\hline
\end{tabular}

Los resultados son expresados en $\mathrm{g} / 16 \mathrm{gN}$

Análisis efectuado en el Laboratorio del Profesor Dr. J. Menden, del Instituto de Nutrición N.$^{\circ}$ 1, Universidad Justus Liebig - Giessen - República Federal Alemana. 
será en el desnutrido proteico-calórico y en la alimentación normal del niño preescolar y escolar.(11-14)

- En todos los pacientes hubo valores iniciales bajos de proteína sanguínea, a expensas de Albúmina con cifras de Globulinas normales. Al final de la experiencia llegaron a valores normales. Es necesario señalar que las Hipoalbuminemias no eran extremas, como se ve en el Kwashiorkor grave.

- Un indicador bioquímico de interés en la evaluación del Estado Nutritivo es el valor de la Creatinina Urinaria. Se sabe que la excreción urinaria refleja fielmente la masa muscular presente, debido al recambio normal de aminoácidos corporales. En las determinaciones de 24 horas se superan las limitaciones de ingesta y las fluctuaciones fisiológicas horarias.

- Con la finalidad de comparar los datos se sugiere correlacionar con la talla y este índice (Creatinina/Talla), sería una expresión directa de la musculatura esquelética.(28)

En todos nuestros pacientes se observa un franco aumento del Indice, lo que revela un aumento de la masa muscular. No disponemos de Tablas para estas edades, por lo que nos pareció más interesante ver la variación de cada caso consigo mismo.

En cuanto a la Talla, no se pueden obtener conclusiones, por ser este parámetro de más lenta recuperación en la desnutrición calórica que el peso. En los niños mayores se considera mejor indicador la relación Peso/Talla, por ser la talla pequeña a veces secuela nutricional y en otros casos talla baja genética.

- Las cifras de Inmunoglobulinas nos presentan alteraciones fundamentales; esta situación no debe extrañar, por cuanto es sabido que en la desnutrición calórico-proteica se alteran mucho más la respuesta inmune específica y la inmunidad celular (Linfocitos T). Se ha descrito aumento de IgE y disminución de IgA secretoria. En la desnutrición proteico-calórica, las Igs pueden estar aumentadas por estímulos antigénicos (infecciones) a repetición.

- El pequeño aumento en relación a la cifra inicial puede explicarse por el aumento de la matriz proteica basal.

- En siete de nuestros pacientes hubo franca mejoría de las cifras de Hemoglobina y Hematocrito, siendo los valores basales menores a $10 \mathrm{~g}$. de Hemoglobina en tres pacientes. En el paciente $\mathrm{N} .{ }^{\circ} 4$, probablemente hubo un factor previo -infeccioso - no detectado o una falta de aporte de $\mathrm{Fe}$ exógeno. En este tipo de desnutrición, no es la anemia hipocroma el rasgo más característico. Varios de nuestros pacientes cercanos a los tres meses presentaban un descenso normal de estos valores ("anemia fisiológica") y su corrección corresponde a la elevación normal de estos índices en todo lactante con buen aporte exógeno de fierro.

- Finalmente queremos señalar que la elaboración y utilización de este producto representa un intento por aprovechar elementos de origen local, con todas las implicancias de tipo económico y social correspondientes, lo que está avalado por las recomendaciones de los expertos en proteínas de FAO/UNICEF, quienes sugieren la elaboración de mezclas proteicas regionales.

- En la búsqueda a nivel mundial de nuevas fuentes nutricias, utilizando recursos de origen vegetal -proteínas mixtas complementadas-, creemos haber contribuido en alguna medida al aporte de soluciones para el problema del hambre, que cada vez afecta a más habitantes del planeta y que en la infancia adquiere un impacto dramático y doloroso.

\section{RESUMEN}

- Entre septiembre de 1977 y abril de 1978 se alimentan diez lactantes menores portadores de Desnutrición Calórico-Proteica de II y III grado, con una fórmula láctea enriquecida con producto farináceo mixto en base a lupino dulce.

- Esta fórmula permite aumentar calorías y proteínas deficitarias, manteniendo un volumen adecuado.

- En todos los lactantes se aprecia excelente aceptabilidad y tolerancia y buena recuperación nutricional.

- Los parámetros químicos y bioquímicos no revelan alteraciones funcionales en higado o riñón (Transaminasas-Urea).

- Los indicadores bioquímicos del estado nutritivo muestran franca corrección (Hemoglobina - Hematocrito - Proteinemia - Creatinina - Inmunoglobulinas).

- Se analiza la aplicación de este producto en la alimentación de niños normales y desnutridos en diferentes grupos etarios.

- Se enfatiza la importancia económico-social del uso de ingredientes de origen local en la manufactura de este producto farináceo.

Se agradece sinceramente al Profesor Dr. Hans Diedrich Cremer, del Instituto de Nutrición de la Universidad de Giessen, República Federal Alemana, por sus valiosas sugerencias en la realización de este estudio y al Ingeniero Agrónomo E. von Baer, de Campex Experimental V.B., de Gorbea, por su colaboración en la manufactura del Producto.

\section{REFERENCIAS}

1 Nutrition, the nervous system and behavior. OPS-OMS, 1972.

2 Puffer, R. R., y Serrano, C. V. Características de la mortalidad en la ninez. OPS-OMS, 1973. 


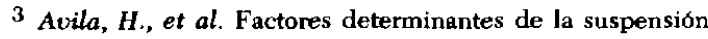
de la lactancia en el grupo de población urbana. Bol of Sanit Panam 84 (5), 1978.

4 Torres, $J$, et al. Alimentación del niño normal y desnutrido. Publ. Doc. 669, 1974. U. de Chile, Sede Norte.

5 Maccioni, A. Tratamiento de la desnutrición. Rev. Ch. Ped. 45: $171,1974$.

6 Fomon, S. Nutrición infantil. México, Interamericana, 1976.

7 Desnutrición infantil. Publ. Doc. 665, 1974. U. de Chile, Facultad de Medicina, Sede Norte.

8 Maccioni, A. Requerimientos de nutrientes. Rev. Ch. Ped. $43(3-4): 25-30,1972$.

9 Mönckeberg, $F$. Requerimientos nutritivos del lactante. Rev. Ch. Ped. 43 (1-2): 7-16, 1972.

10 Note on Human Testing of Supplementary Food Mixtures Nutrition Document WHO-FAO/Unicef, 1966.

11 Desarrollo de una fómula alimentaria para preescolares (Fortesán). INTA-Public. Docente, 1, Stgo., Chile, 1973.

12 Tagle, M. A. "Nutrición 73". Santiago, 1973.

13 Donoso, G. Calorías y proteinas en la alimentación infantil. Nutrición, U. de Chile, 1970.

14 Jellife, D. Nutrición infantil en paises en desarrollo. México, 1974.

15 Human Protein Requirements and their Fullgiment in Practice. Proceed. FAO-WHO, pp. 165, 1955.

16 Von Baer, E. El lupino dulce. Simiente 42: 20-4, 1972.

17 Die lupinen, ein Bertrag zur Nahrungsversergung in den Anden. Emaehrungphysiologische. S. Ernaehrung 15: $391-5,1976$.

18 Gross, R, y Baer, E. V. Posibilidades del Lupinus Mutabilis y Lupinus Albus en los paises andinos. Arch. Latinoam. Nutr. 451-72, 1978 .

19 Cornejo, S., y otros. Lupino como recurso proteico en nutrición animal. En: Situación, análisis y perspectivas del lupino en Chile. Reunión de trabajo organizada por Fundación Chile. 1-2 Dic. 1977.

20 Lupino y quinoa. Estado actual de los conocimientos y de las investigaciones sobre su empleo en alimentación hu- mana. Laboratorio de Bio-Ingenieria, U. de Concepción, Chile, 1975.

21 Neumann, A. M. Enriquecimiento de la harina de trigo con harina de lupino dulce para consumo humano de una población adulta. En: Situación, Análisis y Perspectivas del lupino en Chile. Reunión de trabajo de la Fundación Chile, pp. 89,1978 .

22 Estudio sobre composición química, valor nutritivo y toxicidad en la rata de dos especies de lupino cultivados en Chile. En: Situación, Análisis y Perspectivas del lupino en Chile. Reunión de trabajo Fundación Chile, pp. 97, 1978.

23 Hudson, B. J. F., et al. Lupin: an Arable Food Crop for Temperate Climates. Plant Foods for Man. 2: 81-90, 1976.

24 Eckardt, W. R, et al. Lupinen eine neue alfrucht für Südamerika. Z. Leben-Forsch, 155: 92, 1974.

25 Mermoud, J., y col. Estudio de incorporación de harina de Lupinus Albus en la alimentación normal de un grupo humano. En: Situación, Análisis y Perspectivas del lupino en Chile. Fundación Chile, 1978.

26 Normas de Pediatria. Ministerio de Salud Pública. Chile, 1976.

27 Sempe, M., y col. Tablas de crecimiento de talla y pesos en niños y niñas de 0 a 6 años. Paris, 1971.

28 Viten, E. F., y otros. Métodos de evaluación nutricional proteínico-calóricas en preescolares de condiciones socioeconómicas diferentes. Inst. Nutr. Centroamérica y Panamá (INCAP), Guatemala CA. Arch. Latinoam. Nutr. 23 (1), Mar, 1973.

29 Suskind, R. M. Malnutrition and the Immune Response. Raven Press, New York, 1977.

30 Tabla de composición química de alimentos chilenos. Facultad de Ciencias Químicas, Sede Stgo. Norte, 1975.

31 Valverde, V., y otros. Revision del aporte calórico proternico de las dietas de poblaciones de bajo nivel socioeconómico en Centroamérica. ¿Existe un problema de proteínas? 25 (2), Dic. 1975.

32 Séptimo informe del PAG - FAO - OMS. Roma, 1966, pp. 11-72 y $28-\mathbf{t} 8$.

33 Octavo informe del Comité Mixto de Expertos en Nutrición FAO/OMS, Ginebra, Suiza, XI-70 Roma, 1972, pp. 36-87. 